\title{
Smoking and Mental IIIness: A Bibliometric Analysis of Research Output Over Time
}

\author{
Alexandra P. Metse BPsych (Hons) 1,2, John H. Wiggers PhD2,3,4, \\ Paula M. Wye PhD ${ }^{1,2,3}$, Luke Wolfenden PhD 2,3,4, Judith J. Prochaska PhD, \\ Emily A. Stockings PhD ${ }^{6}$, Jill M. Williams MD, Kerryn Ansell BA (Psych) ${ }^{1}$, \\ Caitlin Fehily BPsych (Hons) 1,2, Jenny A. Bowman PhD ${ }^{1,2}$
}

'School of Psychology, University of Newcastle, Callaghan, Australia; ${ }^{2}$ Hunter Medical Research Institute, New Lambton Heights, Australia; ${ }^{3}$ Hunter New England Population Health, Wallsend, Australia; ${ }^{4}$ School of Medicine and Public Health, University of Newcastle, Callaghan, Australia; ${ }^{5}$ Stanford Prevention Research Center, Department of Medicine, Stanford University, Stanford, CA; ${ }^{6}$ National Drug and Alcohol Research Centre, University of New South Wales, Randwick, Australia; ${ }^{7 R o b e r t ~ W o o d ~ J o h n s o n ~ M e d i c a l ~ S c h o o l, ~ R u t g e r s ~ U n i v e r s i t y, ~ N e w ~ B r u n s w i c k, ~ N J ~}$

Corresponding Author: Alexandra P. Metse, BPsych (Hons), University of Newcastle, University Drive, Callaghan, NSW 2308, Australia. Telephone: 61-(02)-49-217-881; Fax: 61-(02)-49-216-980; E-mail: alexandra.metse@uon.edu.au

\begin{abstract}
Introduction: The prevalence of smoking among persons with a mental illness has remained unchanged, being 2-3 times higher than the general population in high-income countries. Assessment of the volume and characteristics of research output over time can assist in identifying research priorities to promote progress within a field. The aim of this study was to undertake such an assessment in the field of smoking and mental illness.

Methods: A descriptive repeat cross-sectional study was conducted of peer-reviewed publications in Medline and PsycINFO for the periods 1993-1995, 2003-2005, and 2013-2015. Publications were classified as data- or non-data-based; data-based publications were further categorized by study type, population, setting, and for intervention-focused publications by level of evidence and research translation phase.

Results: Included were 547 articles published in 1993-1995 ( $n=65), 2003-2005$ ( $n=153$ ), and 2013-2015 ( $n=329$ ). The number and proportion of data-based publications significantly increased over time, although their focus remained predominantly descriptive ( $\geq 83 \%)$; less than $14 \%$ of publications in any period had an intervention focus. The proportion of publications reporting on study populations with multiple diagnostic categories and recruiting from nonmental health settings, significantly increased from 1993-1995 to 2003-2005, however then plateaued by 2013-2015. The level of evidence provided by intervention-focused publications was suggested to increase over time, however there was no evident variation in translation phase.

Conclusions: Research has increased over time to characterize smoking among those with a mental illness; however more is needed to inform the development and implementation of effective cessation interventions for this group.

Implications: This is the first study to examine the volume and characteristics of research publications in the field of smoking and mental illness over time. The number of publications increased fivefold between 1993-1995 and 2013-2015. Between 1993-1995 and 2003-2005, progression was also indicated by increased: data-based publications, diagnostic diversity of samples, and variation
\end{abstract}


in study settings; however further increases in such measures were not evident in 2013-2015. Notably, it continues to be the case that few intervention studies are undertaken. To achieve meaningful changes in the smoking prevalence of this group, a greater focus on research that assesses the effectiveness and implementation of tailored cessation interventions is required.

\section{Introduction}

Smoking remains the leading cause of preventable illness and death in Australia and internationally. ${ }^{1-3}$ While the prevalence of smoking in the general population has steadily declined to between $15 \%$ and $20 \%$ in high-income countries such as Australia, the United States, and the United Kingdom ${ }^{4-7}$; it has remained largely unchanged for persons with a mental illness, and is currently 2-3 times higher than the general population in such countries. ${ }^{8-11}$ Compared to smokers from the general population, people with a mental illness smoke a greater number of cigarettes, have higher levels of nicotine dependence, ${ }^{9,12}$ and have more difficulty quitting. ${ }^{9}$ Consequently, this population group experience a disproportionate burden of disease associated with tobacco use, including a reduced life expectancy of between 14 and 25 years. ${ }^{13,14}$

Despite a growing recognition of the need to address the high prevalence of smoking among those with a mental illness, ${ }^{15-17}$ its intransigence to change in high-income nations ${ }^{15,18-20}$ suggests a failure of tobacco control initiatives to yield improvements for this group of smokers. ${ }^{21,22}$ As a consequence, there is a need for research to determine effective intervention strategies to reduce smoking and its burden for this disadvantaged group. ${ }^{15}$

Broadly, across public health research there has been recognition of the need for the focus of research to progress over time such that its findings are increasingly likely to be translated into policy and practice, and hence, to effect improvement in health outcomes at a population level. ${ }^{23-25} \mathrm{~A}$ number of frameworks have been suggested for how such progress might be conceptualized or measured, including the Sequential Model proposed by Nutbeam and colleagues $^{24,25}$ and Flay's Eight Phases of Research. ${ }^{26}$ It has been proposed that over time there needs to be an increasing focus on data-based studies (collecting new information) as opposed to nondata-based studies ${ }^{27-30}$; and on intervention research as opposed to descriptive research. ${ }^{23,27-30}$ Further, it has been suggested that intervention-focused research should increasingly utilize research designs that provide high-level evidence (including randomized controlled trials [RCTs] and systematic reviews), ${ }^{23,28}$ and move beyond examining efficacy under optimal or controlled conditions to examining effectiveness and implementation in new populations and settings under real-world conditions. ${ }^{23-25,31,32}$ One recently proposed framework has conceptualized this progression of research as occurring over five phases of research translation: T0 to T4. ${ }^{33-35}$ Stages T0 and T1 involve descriptive research that may inform the development of interventions; T2 involves research that evaluates intervention efficacy; while T3 and T4 reflect the further progression of intervention research to addressing questions of implementation in real world practice and to the assessment of population health impact. ${ }^{33-35}$

Bibliometric analysis represents one method by which the progression of a research field can be measured. ${ }^{23,36}$ Such analyses have been used to critically examine the progression of research in many fields including Indigenous ${ }^{28}$ and rural $^{37}$ health, alcohol misuse, ${ }^{27}$ physical activity and falls prevention, ${ }^{23}$ and cancer. ${ }^{30,38-40}$ The outcomes of a number of such studies have demonstrated a lack of progression over time, concluding that this may contribute to a lack of impact on public health outcomes. ${ }^{23,27,40}$ For example, no change in the proportion of data-based and non-data-based research surrounding the delivery of multidisciplinary cancer care was reported between 1997-2001 and 2002-2006..$^{40}$ With respect to research in the field of physical activity between 1988-1989 and 2008-2009, little variation in the proportion of intervention research being undertaken was noted, ${ }^{23}$ and a persistent predominance of efficacy, relative to replication and dissemination trials was evident. ${ }^{23}$

Few bibliometric studies have been conducted in the field of smoking research. Sanson-Fisher et al. ${ }^{29}$ reported a bibliometric analysis of "public health" smoking research (that which is nonlaboratory and nonclinical) being undertaken across three decades (1987-1988, 1997-1998, 2005-2006) and found that while the proportion of data-based research increased in 2005-2006, 78\% of publications remained descriptive. Another bibliometric analysis of smoking research focused on smoking and pregnancy; with a particular focus on the volume and country of origin of research, and the gender of the researchers. This review found that between 1900 and 2012, the volume of annual publications increased from 35 to approximately 700 ; the majority of research was undertaken in the United States (35\%) followed by the United Kingdom $(10 \%)$; and that researchers were predominantly female $(55 \%) .{ }^{41}$ Neither of these previous bibliometric reviews ${ }^{29,41}$ considered the diversity of study populations or settings utilized in included publications, or any other similar indicators of research progression. ${ }^{32}$

Only one bibliometric analysis has investigated the volume and characteristics of research in the field of smoking and mental illness, and this was limited to Australian research published since January 2000 regarding tobacco use in the context of co-occurring physical illnesses (such as cardiovascular disease). ${ }^{42}$ Included publications were identified from a Google Scholar search $(n=182)$ and classified into one or more of 11 domains, and analyses were limited to identifying how many studies were conducted where "tobacco smoking was the major topic." The authors concluded that smoking was the major topic for $19 \%$ of the publications, but reported no further assessment of the characteristics of such publications or of change over time. This study focused exclusively on persons with a "severe mental illness," thus excluding a large proportion of the population of smokers with a mental illness. Further, by limiting the study to specific diagnostic groups, and not considering the study settings, an indication of outcome generalizability and translational utility could not be ascertained..$^{32,43}$ Thus, there is a key gap in the literature examining research outputs among smokers with a mental illness. Examining change in research outputs in this field is particularly important given recent high-profile publications identifying smoking and mental illness as a priority area for increased research attention. ${ }^{20,21,44,45}$

The current study was conducted to describe the volume and characteristics (data-based, study type, study population, and study setting) of research publications in the field of smoking and mental illness across three time periods. For intervention-focused research, the level of evidence and phase of research translation were also considered; as indications of progress in the quality of research and the potential for population level impact respectively. 


\section{Methods}

\section{Design}

A descriptive repeat cross-sectional study was conducted of peerreviewed publications.

\section{Data Sources}

Medline and PsycINFO databases were used to locate peer-reviewed publications relating to smoking and mental illness for the periods 1993-1995, 2003-2005, and 2013-2015: the objective being to systematically assess trends in study characteristics over time for a sample of publications reporting on smoking and mental illness, rather than the absolute output of publications for the specified periods. Medline and PsycINFO were utilized as they index the majority of peer-reviewed journals publishing public health and related research, and both databases adopt similar search strategies and platformsenabling almost identical search strategies to be undertaken. In both databases, the advanced search option was used. "Keyword" and "map term to subject heading" options were selected. The search terms and procedures are outlined in Supplementary Figure 1. Final searches for both "smoking" and "mental illness" were combined using the "AND" function and limited to those published in English and by year (1993-1995, 2003-2005, and 2013-2015) to obtain the final search results.

\section{Inclusion and Exclusion Criteria}

Data and non-data-based publications were included. Data-based publications were those reporting original data or results, or systematically synthesizing existing results. Such publications were included if (1) the sample consisted of persons with an identified mental health issue (current or past) and (2) quantitative and/or qualitative data surrounding smoking or related behaviors of such samples were presented. Publications using nonhuman samples were excluded.

Non-data-based publications (those not reporting data or systematically synthesizing results) were included if the topic of the paper directly related to smoking and mental illness. Examples of such publications included study protocols, letters to the editor, commentaries, and nonsystematic literature reviews.

\section{Screening and Data Extraction}

One author (CF) and a research assistant screened publications for inclusion using the title, abstract and where necessary, full text. Data were extracted from included publications according to the data classifications outlined below. To provide a measure of quality control, another author (APM) extracted data for a randomly selected subsample $(15 \%)$ of publications; with complete agreement achieved between reviewers across all classifications.

\section{Publication Classification}

All included publications were categorized as either data-based or non-data-based. Data-based publications were further classified according to: study type, study population and study setting. No additional classification was undertaken for non-data-based publications.

\section{Data-Based Papers}

Study Type. To determine the focus of each data-based publication, such publications were categorized, based on a classification commonly adopted in other bibliometric reviews, ${ }^{23,27-30}$ as either:
- Measures: publications that examined the quality (validity or reliability) or acceptability of a measurement instrument used to assess smoking or a related factor/characteristic.

- Descriptive: publications that reported data related to smoking and mental illness, but did not examine the efficacy or effectiveness of a proposed intervention to address smoking.

- Intervention: publications that reported the outcomes of any intervention, or a group of interventions, aimed at addressing smoking. All commonly utilized outcome variables in trials relating to smoking were considered relevant: uptake of smoking, smoking cessation/abstinence, number of cigarettes smoked, nicotine dependence, quit attempts, readiness/motivation to quit, interest in quitting and provision of smoking-related care.

Publications classified as having an intervention focus were further categorized in terms of the "level of evidence" (assessed based on study design: systematic reviews/meta-analyses, RCTs, other controlled trials, or other), ${ }^{46,47}$ and "translation phase" (phases: T2, T3, T4). ${ }^{33-35,48}$

Study Population. The population of people included in each study was categorized according to the following diagnostic groups: (1) "severe" disorders (a diagnosis of schizophrenia and related psychosis, bipolar disorder and/or major depressive disorder $)^{49-52}$; (2) substance use disorders; or (3) an "other" disorder (persons with a mental health disorder/s that is not category 1 or 2 ). Studies consisting of participants with more than one type of mental illness were assigned to all relevant categories. Substance use disorders were classified separately due to the literature reflecting some lack of consensus regarding their status as mental illness. ${ }^{15}$

Study Setting. The study setting was recorded as either: mental health inpatient, mental health outpatient or community based, generalist health care (including general practitioners), or "other" (including web or media based, universities, or sub group analyses from population surveys).

\section{Data Analysis}

Data were analyzed using SPSS Statistics version $22 .{ }^{53}$ Descriptive analysis was used to report on the total number of publications, and the number and proportion of publications within each of the classifications (study type, study population, and study setting), and sub classifications for intervention publications (level of evidence and research translation phase) across the three time periods.

Chi-square or Fisher's exact tests, where applicable, were used to assess for change in the volume and characteristics of publications over time. Where significant changes were evident, standardized residuals for each time period were examined, with scores of greater than or equal to \pm 1.96 indicating cells with significant variation from expected. ${ }^{54}$ For such analyses, study type was reduced to measures/descriptive and intervention research. Two variables were created for study population: "severe" only versus all other categories; and >1 category versus 1 category only. For study setting, mental health inpatient and mental health outpatient/ community settings were combined to form one category, with generalist health settings, other settings and those using a combination of mental and nonmental health settings combined to form a second. With respect to intervention-focused research, level of evidence was reduced to three levels (other controlled trials/other, RCTs, and systematic reviews/meta-analyses) and research translation phase to two (T2, T3/T4). 


\section{Results}

\section{Included Publications}

Figure 1 illustrates the flow diagram for publications identified in the review. Two-thousand forty-one publications were identified across the two databases. Two-hundred twenty-three duplicates were removed, leaving 1818 to be screened for inclusion. Five-hundred forty-seven publications were included in the review.

\section{Volume of Data-Based Publications}

The total number of publications relating to smoking and mental illness over the three time periods was 547; 65 (12\%) in 1993 $1995,153(28 \%)$ in 2003-2005, and $329(60 \%)$ in 2013-2015 (Table 1). The proportion of data-based publications, relative to non-data-based publications significantly increased from $62 \%$ $(n=40)$ in $1993-1995$ to $76 \%(n=116)$ in $2003-2005$, however no further increase was evident in $2013-2015(77 \%, n=252)\left(\chi^{2}(2\right.$, $N=547)=6.66, p=.04)$.

\section{Study Type}

In 1993-1995, 83\% ( $n=33)$ of data-based publications were categorized as descriptive, $5 \%(n=2)$ as measures, and $13 \%(n=5)$ as intervention. In 2003-2005 and 2013-2015, 89\% $(n=103)$ and $86 \%(n=217)$ of publications were classified as descriptive respectively, $2 \%(n=2)$ and $1 \%(n=2)$ as measures, and $9 \%(n=11)$ and $13 \%(n=33)$ as intervention research, with no significant difference by study type across the three time periods $(p=.64 ;$ Table 1$)$.

\section{Level of Evidence}

Among the intervention-based publications, the proportion of systematic reviews/meta-analysis-based publications was $0 \%(n=0)$ in 1993-1995, 9\% $(n=1)$ in 2003-2005 and 15\% $(n=5)$ in 20132015 (Table 2). RCT designs were adopted for $20 \%(n=1)$ of intervention research in 1993-1995, 18\% $(n=2)$ in 2003-2005 and 55\% $(n=18)$ in 2013-2015, with a borderline significant increasing trend across the three time periods $(p=.06)$.

\section{Research Translation Phase}

There was little variation in the research translation phase of intervention-focused publications across the three time periods $(p=1.00)$ : all were classified as T2 in 1993-1995, with identical proportions classified as T2 (91\%) and T3 (9\%) in both 2003-2005 and 2013-2015 (Table 2).

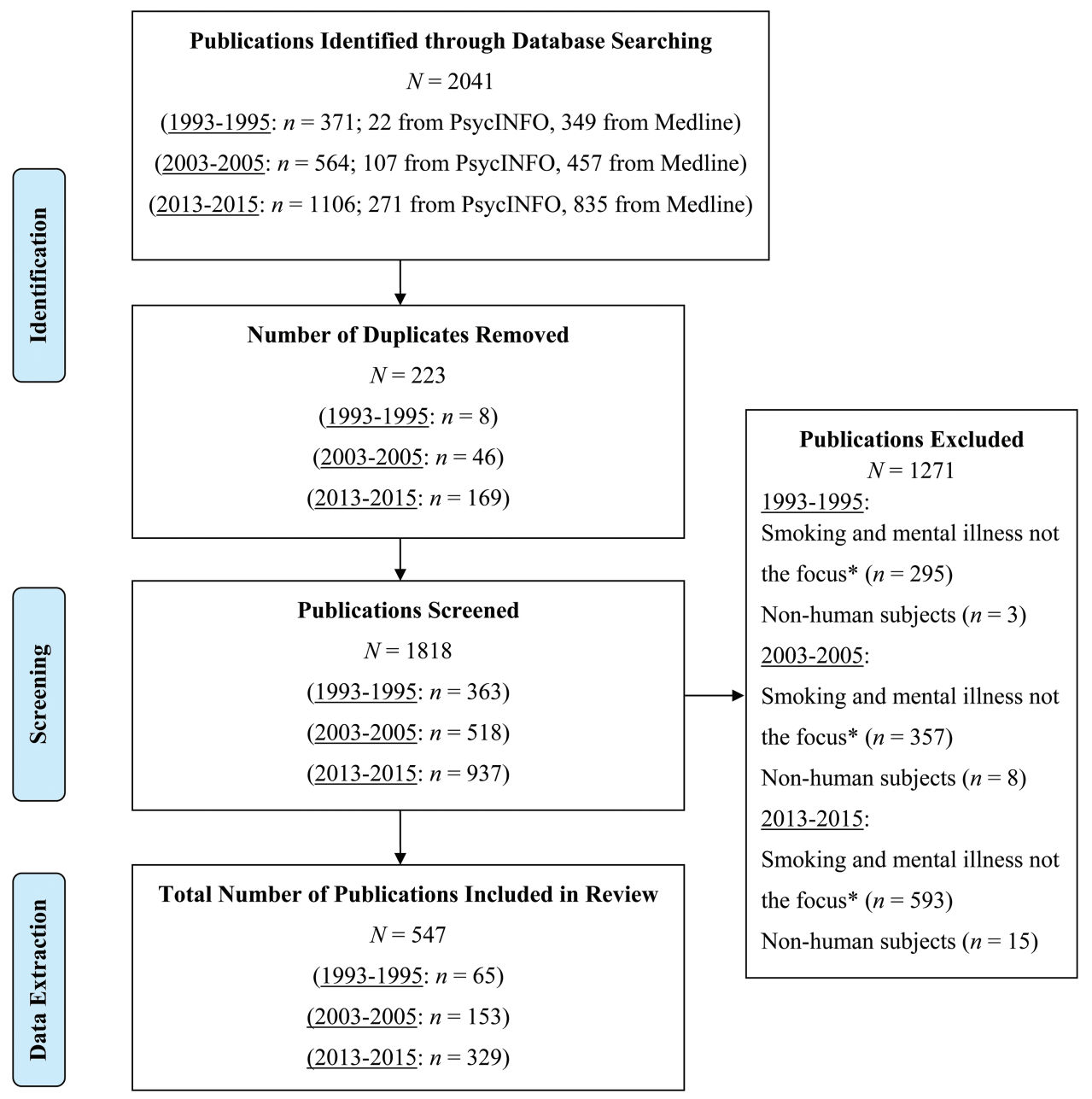

Figure 1. Flow diagram for inclusion of articles in the review. *Publications that made no mention in the aims nor included any significant analysis or discussion surrounding smoking and mental illness were not included. Note. References for publications included in the review can be sourced from Supplementary Figure 2. 
Table 1. Total Number of Publications Related to Smoking and Mental Illness Between 1993-1995, 2003-2005, and 2013-2015, According to Study Type

\begin{tabular}{lccccc}
\hline & $1993-1995$ & & $2003-2005$ & & $2013-2015$ \\
\cline { 2 - 2 } \cline { 5 - 6 } \cline { 5 - 6 } & $n(\%)$ & & $n(\%)$ & & $n(\%)$ \\
\hline Non-data-based research & $25(38)$ & & $37(24)$ & & $77(23)$ \\
Data-based research & $40(62)$ & & $116(76)$ & & $252(77)$ \\
Descriptive & $33(83)$ & & $103(89)$ & & $217(86)^{\mathrm{a}}$ \\
Measures & $2(5)$ & & $2(2)$ & & $2(1)$ \\
Intervention & $5(13)^{\mathrm{b}}$ & & $11(9)$ & & $33(13)$ \\
\hline
\end{tabular}

${ }^{a}$ Five publications in this category were systematic reviews not relating to intervention efficacy/effectiveness.

${ }^{b}$ Three publications in this category originated from a single intervention study, whereas in subsequent years all intervention-focused publications were based on discrete studies.

Table 2. Level of Evidence and Research Translation Phase for Intervention Publications Between 1993-1995, 2003-2005, and 2013-2015

\begin{tabular}{|c|c|c|c|}
\hline & \multirow{2}{*}{$\frac{1993-1995}{n(\%)}$} & \multirow{2}{*}{$\frac{2003-2005}{n(\%)}$} & \multirow{2}{*}{$\frac{2013-2015}{n(\%)}$} \\
\hline & & & \\
\hline \multicolumn{4}{|l|}{ Level of evidence } \\
\hline $\begin{array}{l}\text { Meta-analyses/ systematic } \\
\text { reviews }\end{array}$ & $0(0)$ & $1(9)$ & $5(15)$ \\
\hline RCT & $1(20)$ & $2(18)$ & $18(55)$ \\
\hline Other controlled trial & $2(40)$ & $3(27)$ & $3(9)$ \\
\hline Other & $2(40)$ & $5(45)$ & $7(21)$ \\
\hline \multicolumn{4}{|l|}{ Research translation phase } \\
\hline $\mathrm{T} 2$ & $5(100)$ & $10(91)$ & $30(91)$ \\
\hline T3 & $0(0)$ & $1(9)$ & $3(9)$ \\
\hline $\mathrm{T} 4$ & $0(0)$ & $0(0)$ & $0(0)$ \\
\hline
\end{tabular}

RCT = randomized controlled trial. T2 research evaluates intervention efficacy, while T3 research assesses intervention effectiveness and addresses questions of implementation in real world practice. Research in T4 assesses the population health impact of an intervention.

\section{Study Population}

Table 3 describes the number and proportion of publications reporting on the identified diagnostic categories across the three time periods. The proportion of publications focused on only severe mental disorders was $8 \%(n=3)$ in $1993-1995,18 \%(n=21)$ in $2003-2005$ and $20 \%(n=50)$ in 2013-2015, with no statistically significant change across the three periods $\left(\chi^{2}(2, N=408)=0.17, p=.17\right)$.

The proportion of publications reporting on more than one of the specified diagnostic categories significantly increased from $28 \%(n=11)$ in $1993-1995$ to $56 \%(n=65)$ in $2003-2005$, however remained similar in $2013-2015(57 \%, n=144)\left(\chi^{2}(2\right.$, $N=408)=12.50, p=.002$ ). Of the publications focusing on only one of the diagnostic classifications, $75 \%$ concentrated on substance use disorders in 1993-1995, while severe mental disorders was the focus for nearly half (41\% and 46\%) in 2003-2005 and 2013-2015.

\section{Study Setting}

The number and proportion of publications undertaken across the specified settings are reported for the three time periods in Table 3. The proportion of publications undertaken in mental health inpatient facilities was $43 \%(n=17)$ in $1993-1995,16 \%(n=19)$
Table 3. Study Population and Setting for Data-Based Publications Between 1993-1995, 2003-2005, and 2013-2015

\begin{tabular}{|c|c|c|c|}
\hline & 1993-1995 & 2003-2005 & 2013-2015 \\
\hline & $n(\%)$ & $n(\%)$ & $n(\%)$ \\
\hline \multicolumn{4}{|l|}{ Study population } \\
\hline Severe ${ }^{a}$ disorders & $3(8)$ & $21(18)$ & $50(20)$ \\
\hline Substance use disorders & $22(55)$ & $17(15)$ & $24(10)$ \\
\hline Other disorders & $4(10)$ & $13(11)$ & $34(13)$ \\
\hline Severe + other & $2(5)$ & $14(12)$ & $43(17)$ \\
\hline $\begin{array}{l}\text { Severe and/or other }+ \\
\text { substance use disorders }\end{array}$ & $8(20)$ & $49(42)$ & $96(38)$ \\
\hline Unclear/ not specified & $1(3)$ & $2(2)$ & $5(2)$ \\
\hline \multicolumn{4}{|l|}{ Study setting } \\
\hline Mental health inpatient & $17(43)$ & $19(16)$ & $37(15)$ \\
\hline $\begin{array}{l}\text { Mental health outpatient or } \\
\text { community based }\end{array}$ & $8(20)$ & $27(23)$ & $65(26)$ \\
\hline Generalist health care & $1(3)$ & $5(4)$ & $9(4)$ \\
\hline Other & $9(23)$ & $57(49)$ & $121(48)$ \\
\hline $\begin{array}{l}\text { Mental health inpatient + } \\
\text { outpatient/ community }\end{array}$ & $5(13)$ & $7(6)$ & $10(4)$ \\
\hline $\begin{array}{l}\text { Mental health outpatient/ } \\
\text { community + other }\end{array}$ & $0(0)$ & $1(1)$ & $6(2)$ \\
\hline$>3$ settings & $0(0)$ & $0(0)$ & $4(2)$ \\
\hline
\end{tabular}

${ }^{a}$ Schizophrenia, bipolar disorder, and/or major depressive disorder.

in 2003-2005 and 15\% $(n=37)$ in 2013-2015; while the proportion utilizing outpatient or community mental health settings was $20 \%(n=8)$ in $1993-1995,23 \%(n=27)$ in $2003-2005$ and $26 \%$ $(n=65)$ in 2013-2015. "Other" settings were used for 23\% $(n=9)$ of publications in 1993-1995, 49\% $(n=57)$ in 2003-2005 and $48 \%$ $(n=121)$ in $2013-2015$. Relative to mental health specific settings exclusively, the use of generalist health care and/or other settings significantly increased from $26 \%$ in $1993-1995$ to $53 \%$ in $2003-2005$, however no further increase was evident in $2013-2015$ (52\%) ( $\chi^{2}(2$, $N=408)=15.47, p<.001)$.

\section{Discussion}

The current study is novel in determining the volume and characteristics of research publications across three time periods, via bibliometric analysis, in the field of smoking and mental illness, and fills a key gap in the literature. Overall, the total number of studies reporting on smoking and mental illness increased fivefold from 65 in 1993-1995 to 329 in 2013-2015; an increase that was also observed in the number and proportion of data-based publications. There was no variation in the proportion of study types across the time periods, with research remaining largely descriptive, with limited focus on intervention and measures. For intervention-focused research, there was suggestion of an increase in the adoption of RCT study designs in the most recent period, however the research translation phase remained unchanged over time. Between 1993-1995 and 2003-2005, study populations became more diagnostically heterogeneous, and there was an increased focus on settings other than mental health care specific facilities; however no further increase in such measures were evident in 2013-2015.

As proposed by models of research translation, ${ }^{23-25}$ the progression of research within a field from describing health issues to the development and assessment of interventions, and, where applicable, their dissemination is required to achieve a population health 
impact. The sequential model proposed by Nutbeam and others ${ }^{24,25}$ suggests that such progression requires at least some increase in the relative amount of intervention-focused publications over time. Such an increase was reported by Sanson-Fisher and colleagues ${ }^{29}$ in the field of smoking and public health generally between 1987 and 2006. In contrast, the unchanging predominance of descriptive research in the field of smoking and mental illness between the periods compared suggests that such progression has not occurred. While some descriptive research will continue to be important in this field, including causal inference studies which help progress understanding of the relationship between smoking and mental illness, the balance of research effort nevertheless needs to shift towards intervention research given the need to reduce the prevalence of smoking and its negative consequences for people with a mental illness.

It has been suggested in other fields of research that the predominance of descriptive research may be associated with advantages it offers for researchers in terms of greater convenience, productivity, and lower cost..$^{23,28-30}$ Possible strategies to promote a greater focus on intervention research may include for instance such research being weighted more favorably by journal editors and/or in academic promotion criteria. ${ }^{55}$ Further, it might be speculated that a lack of policy-driven or translation-focused research funding, in comparison to investigator driven research funding, may have contributed, ${ }^{55}$ or that "smoking and mental illness" may not fit neatly into any one area of funding and so to some extent risk falling into a "funding gap." 21 Some experts in the United States have argued strongly that tobacco use by people with a mental illness continues to be insufficiently prioritized for policy and program attention, and that this population group should be more strongly identified as a "tobacco use disparity group," particularly at a national level. ${ }^{56}$

While there was no difference in the amount of intervention research over time, there was some indication of progression in terms of the level of evidence provided by the intervention research undertaken, with a modest rise in the number and proportion of systematic reviews/meta-analyses and RCTs between 1993-1995 and 2013-2015. The increasing tendency toward synthesizing research findings through systematic review/ meta-analyses perhaps also suggests an increased acknowledgement of the importance of this public health issue. However, the stagnation in translation phasewith 9\% categorized as T3 in both 2003-2005 and 2013-2015 periods-highlights the need for progression from efficacy trials to those assessing the effectiveness and dissemination of interventions.

The greater diversity of study settings and diagnostic heterogeneity of samples observed across the compared periods may suggest an increase in the generalizability and possibly translational utility of findings. ${ }^{32,43}$ Health care settings remained the predominant focus of research across the time periods, particularly so for intervention research. As such settings have been recognized to provide an opportunity to reach and systematically offer cessation support to all patients, ${ }^{57}$ including those with a mental illness, ${ }^{15,58}$ such a focus could be appropriate. ${ }^{22}$ In terms of mental health specific settings, the increased attention on community/ outpatient mental health facilities, relative to inpatient facilities, could be speculated to be a positive reflection of a less exclusive focus on severe diagnoses or patients experiencing acute illness episodes, but may also reflect a change in the delivery of mental health care towards a greater focus on community based services in some countries. ${ }^{59,60}$ In Australia, it has been estimated that $64 \%$ of smokers with a 12 -month mental disorder had not accessed any health services over a period of 1 year. ${ }^{19}$ The increasing focus on settings other than health care settings observed in this study may therefore indicate an increased recognition of the need to address smoking among these community living people with mental illness who do not regularly access the health system. ${ }^{19}$

A notable proportion of publications in the current study were restricted to a sample with only one diagnostic classification, that is, only "severe" or only substances use disorders. Where only one diagnostic classification was included, the large majority of publications in 1993-1995 focused on substance use disorders (76\%), whereas almost half focused on severe mental disorders in both 2003-2005 and 2013-2015. It might be speculated that the predominant attention to substance use disorders in 1993-1995 reflects factor such as an early recognition of the high smoking prevalence among persons with such disorders, or initial concerns over the possibility of adverse events arising from concurrent treatment of tobacco and other substance disorders. The observed shift in focus to severe mental disorders in later periods could be understandable given that the prevalence of smoking among those meeting such criteria has been recognized as particularly high. ${ }^{8,61}$ The increase in the diagnostic heterogeneity of samples over time however, is likely to promote increased generalizability and translational utility of findings. ${ }^{32,43}$

The findings from the current study should be interpreted in the context of a number of methodological characteristics. Publications were sourced from only two research databases and the findings therefore do not represent the total sum of research undertaken, although this is unlikely to have influenced relative trends in publication characteristics across the time periods. Also, the adopted methodology would not have sourced relevant grey literature and government documents, and allows reflection only on the amount and quality of peer-reviewed publications. Future studies assessing publication characteristics across multiple time periods should utilize a greater number of peer-reviewed databases and, although difficult to systemically undertake, attempt to source non peer-reviewed resources to address these potential limitations. Relevant publications may not have been sourced using our search terms if they related to "lifestyle interventions" where smoking was addressed, leading to a possible underestimation of the amount of intervention-focused research. In addition, comparing 3-year intervals across two decades may have introduced a selection bias. Employing broader search terms that include all physical health risk behaviors and comparing decades would be of benefit in future studies. Also, while this study considered phase of research translation for interventionfocused publications, future research might consider the degree to which research across all study types, used large representative samples and therefore had relevance at a population level.

Overall, the total number of studies reporting on smoking and mental illness increased fivefold between 1993-1995 and 20132015. The findings from this study suggest a number of other positive indicators of progression between 1993-1995 and 2003-2005, including increased: proportion of data-based publications, diagnostic diversity of samples, and variation in study settings; however, no further increase in such measures were evident in 2013-2015. Notably, it continues to be the case that few intervention studies appear to be undertaken. To achieve meaningful changes in the smoking prevalence of this group, a greater increase in research volume is required, particularly that which assesses the effectiveness and implementation of tailored cessation interventions.

\section{Supplementary Material}

Supplementary Figures 1 and 2 can be found online at http://www. ntr.oxfordjournals.org 


\section{Funding}

This work was supported by the National Health and Medical Research Council (NHMRC) (grant number: G1100130). EAS is supported by the NHMRC Centre of Research Excellence in Mental Health Systems Improvement (CREMSI) (grant number: 1041131). The National Drug and Alcohol Research Centre at the University of New South Wales, Australia is supported by funding from the Australian Government under the Substance Misuse Prevention and Service Improvements Grant Fund. JJP is supported by the National Institutes of Health (grant number: HL117736-01) and the State of California TobaccoRelated Disease Research Program (grant number: 24RT-0035).

\section{Declaration of Interests}

JJP has consulted to Pfizer, which makes smoking cessation medications, and has been an expert witness for plaintiffs' counsel in lawsuits against the tobacco companies. JMW has received unrestricted continuing medical education grants from Pfizer. The other authors declare they have no competing interests.

\section{Acknowledgments}

The authors would like to acknowledge Kristy Rudd for her assistance identifying search terms and database searching. We would also like to acknowledge Lauren Gibson for her contribution to the publication screening and data extraction process.

\section{References}

1. Lim SS, Vos T, Flaxman AD, et al. A comparative risk assessment of burden of disease and injury attributable to 67 risk factors and risk factor clusters in 21 regions, 1990-2010: a systematic analysis for the Global Burden of Disease Study 2010. Lancet. 2012;380(9859):2224-2260. doi:10.1016/ s0140-6736(12)61766-8.

2. United States Department of Health and Human Services. The Health Consequences of Smoking-50 Years of Progress: A Report of the Surgeon General. Atlanta, GA: U.S. Department of Health and Human Services, Centers for Disease Control and Prevention, National Center for Chronic Disease Prevention and Health Promotion, Office on Smoking and Health; 2014.

3. Allender S, Balakrishnan R, Scarborough P, Webster P, Rayner M. The burden of smoking-related ill health in the UK. Tob Control. 2009;18(4):262267. doi:10.1136/tc.2008.026294.

4. Australian Institute of Health and Welfare. National Drug Strategy Household Survey: Detailed findings. Canberra, Australia: AIHW; 2007. www.aihw.gov.au. Accessed December 12, 2014.

5. Centers for Disease Control. Current cigarette smoking among adults United States, 2011. Morb Mortal Wkly Rep. 2012;61(44):889-894.

6. World Health Organization. Report on the Global Tobacco Epidemic, 2009: Implementing Smoke Free Environments. Geneva, Switzerland: WHO; 2009.

7. Office for National Statistics. Integrated Houselhold Survey, January to December 2013: Experimental Statistics. Newport, UK: Office for National Statistics; 2014. http://webarchive.nationalarchives.gov. uk/20160105160709/http://www.ons.gov.uk/ons/dcp171778_379565.pdf. Accessed March 3, 2015.

8. de Leon J, Diaz FJ. A meta-analysis of worldwide studies demonstrates an association between schizophrenia and tobacco smoking behaviors. Schizophr Res. 2005;76(2-3):135-157. doi:10.1016/j. schres.2005.02.010.

9. Lasser K, Boyd W, Woolhandler S, et al. Smoking and mental illness: a population based study. JAMA. 2000;284(20):606-610. doi:10.1001/ jama.284.20.2606

10. McClave AK, McKnight-Eily LR, Davis SP, Dube SR. Smoking characteristics of adults with selected lifetime mental illnesses: results from the 2007 National Health Interview Survey. Am J Public Health. 2010;100(12):2464-2472. doi:10.2105/ajph.2009.188136.

11. Centers for Disease Control and Prevention. Vital signs: current cigarette smoking among adults aged $\geq 18$ years with mental illness - United States, 2009-2011. Morb Mortal Wkly Rep. 2013;62(5):81-87.

12. Bowden JA, Miller CL, Hiller JE. Smoking and mental illness: a population study in South Australia. Aust N Z J Psychiatry. 2011;45(4):325-331. doi:10.3109/00048674.2010.536904.

13. Colton CW, Manderscheid RW. Congruencies in increased mortality rates, years of potential life lost, and causes of death among public mental health clients in eight states. Prev Chronic Dis. 2006;3(2):A42.

14. Lawrence D, Hancock KJ, Kisely S. The gap in life expectancy from preventable physical illness in psychiatric patients in Western Australia: retrospective analysis of population based registers. BMJ (Clinical Research Ed.). 2013;346(7909):f2539. doi:http://dx.doi.org/10.1136/bmj.f2539

15. Royal College of Physicians, Royal College of Psychiatrists. Smoking and Mental Health. London, UK: RCP Council Report CR1782013; 2013.

16. Smith PH, Mazure CM, McKee SA. Smoking and mental illness in the US population. Tob Control. 2014;23(e2):e147-e153. doi:10.1136/ tobaccocontrol-2013-051466.

17. Gartner C, Hall W. Tobacco harm reduction in people with serious mental illnesses. Lancet Psychiatry. 2015;2(6):485-487. doi:10.1016/ S2215-0366(15)00238-2.

18. Szatkowski L, McNeill A. Diverging trends in smoking behaviors according to mental health status. Nicotine Tob Res. 2015;17(3):356-360. doi:10.1093/ntr/ntu173.

19. Lawrence D, Mitrou F, Zubrick SR. Smoking and mental illness: results from population surveys in Australia and the United States. BMC Public Health. 2009;9:285. doi:10.1186/1471-2458-9-285.

20. Steinberg ML, Williams JM, Li Y. Poor mental health and reduced decline in smoking prevalence. Am J Prev Med. 2015;49(3):362-369. doi:10.1016/j.amepre.2015.01.016.

21. Williams JM, Willett JG, Miller G. Partnership between tobacco control programs and offices of mental health needed to reduce smoking rates in the United States. JAMA Psychiatry. 2013;70(12):1261-1262. doi:10.1001/jamapsychiatry.2013.2182.

22. Britton J. Treating smoking in mental health settings. Lancet Psychiatry. 2015;2(5):364-365. doi:10.1016/S2215-0366(15)00102-9.

23. Milat AJ, Bauman AE, Redman S, Curac N. Public health research outputs from efficacy to dissemination: a bibliometric analysis. BMC Public Health. 2011;11(1):934. doi:10.1186/1471-2458-11-934.

24. Nutbeam D, Smith C, Catford J. Evaluation in health education. A review of progress, possibilities, and problems. J Epidemiol Commun $H$. 1990;44(2):83-89.

25. Nutbeam D. Achieving 'best practice' in health promotion: improving the fit between research and practice. Health Educ Res. 1996;11(3):317-326.

26. Flay BR. Efficacy and effectiveness trials (and other phases of research) in the development of health promotion programs. Prev Med. 1986;15(5):451474. doi:http://dx.doi.org/10.1016/0091-7435(86)90024-1.

27. Shakeshaft AP, Bowman JA, Sanson-Fisher RW. Behavioural alcohol research: new directions or more of the same? Addiction. 1997;92(11):1411-1422.

28. Sanson-Fisher RW, Campbell EM, Perkins JJ, Blunden SV, Davis BB. Indigenous health research: a critical review of outputs over time. Med J Aust. 2006;184(10):502-505.

29. Sanson-Fisher RW, Campbell EM, Htun AT, Bailey LJ, Millar CJ. We are what we do: research outputs of public health. Am J Prev Med. 2008;35(4):380-385. doi: 10.1016/j.amepre.2008.06.039.

30. Bailey LJ, Sanson-Fisher R, Aranda S, et al. Quality of life research: types of publication output over time for cancer patients, a systematic review. Eur J Cancer Care (Engl). 2010;19(5):581-588. doi:10.1111/j.1365-2354.2009.01109.x.

31. Yoong SL, Clinton-McHarg T, Wolfenden L. Systematic reviews examining implementation of research into practice and impact on population health are needed. J Clin Epidemiol. 2015;68(7):788-791. doi:10.1016/j. jclinepi.2014.12.008. 
32. Bauer MS, Damschroder L, Hagedorn H, Smith J, Kilbourne AM. An introduction to implementation science for the non-specialist. BMC Psychol. 2015;3(1):32. doi:10.1186/s40359-015-0089-9.

33. Khoury MJ, Gwinn M, Yoon PW, et al. The continuum of translation research in genomic medicine: how can we accelerate the appropriate integration of human genome discoveries into health care and disease prevention? Genet Med. 2007;9(10):665-674. doi:10.1097GIM.0b013e31815699d0.

34. Khoury MJ, Gwinn M, Ioannidis JP. The emergence of translational epidemiology: from scientific discovery to population health impact. Am J Epidemiol. 2010;172(5):517-524. doi:10.1093/aje/kwq211.

35. Meslin EM, Blasimme A, Cambon-Thomsen A. Mapping the translational science policy 'valley of death'. Clin Transl Med. 2013;2(1):14. doi:10.1186/2001-1326-2-14.

36. Koskinen J, Isohanni $\mathrm{M}$, Paajala $\mathrm{H}$, et al. How to use bibliometric methods in evaluation of scientific research? An example from Finnish schizophrenia research. Nord J Psychiatry. 2008;62(2):136-143. doi:10.1080/08039480801961667.

37. McLean R, Mendis K, Harris B, Canalese J. Retrospective bibliometric review of rural health research: Australia's contribution and other trends. Rural Remote Health. 2007;7(4):767.

38. Sanson-Fisher R, Bailey LJ, Aranda S, et al. Quality of life research: is there a difference in output between the major cancer types? Eur J Cancer Care (Engl). 2010;19(6):714-720. doi:10.1111/j.1365-2354.2009.01158.x.

39. Paul CL, Clinton-McHarg T, Sanson-Fisher RW, Douglas H, Webb G. Are we there yet? The state of the evidence base for guidelines on breaking bad news to cancer patients. Eur J Cancer. 2009;45(17):2960-2966. doi:10.1016/j.ejca.2009.08.013.

40. CareyM,Sanson-FisherR,Lotfi-JamK,SchofieldP,ArandaS.Multidisciplinary care in cancer: do the current research outputs help? Eur J Cancer Care (Engl). 2010;19(4):434-441. doi:10.1111/j.1365-2354.2009.01105.x.

41. Mund M, Kloft B, Bundschuh M, et al. Global research on smoking and pregnancy-a scientometric and gender analysis. Int J Environ Res Public Health. 2014;11(6):5792-5806. doi:10.3390/ijerph110605792.

42. Happell B, Galletly C, Castle D, et al. Scoping review of research in Australia on the co-occurrence of physical and serious mental illness and integrated care. Int J Ment Health Nurs. 2015;24(5):421-438. doi:10.1111/inm.12142.

43. Mercer SL, DeVinney BJ, Fine LJ, Green LW, Dougherty D. Study designs for effectiveness and translation research: identifying trade-offs. Am J Prev Med. 2007;33(2):139-154. doi:10.1016/j.amepre.2007.04.005.

44. Lancet.Smoke alarm: mental illness and tobacco.Lancet.2013;381(9872):1071. doi:10.1016/S0140-6736(13)60677-7.

45. Gurillo P, Jauhar S, Murray RM, MacCabe JH. Does tobacco use cause psychosis? Systematic review and meta-analysis. Lancet Psychiatry. 2015;2(8):718-725. doi:10.1016/S2215-0366(15)00152-2.

46. National Health and Medical Research Council. Assessing the Evidence. Canberra, Australia: Commonwealth of Australia; 2000.

47. Petrisor B, Bhandari M. The hierarchy of evidence: levels and grades of recommendation. Indian J Orthop. 2007;41(1):11-15. doi:10.4103/0019-5413.30519.
48. Gartlehner G, Hansen RA, Nissman D, Lohr KN, Carey TS. A simple and valid tool distinguished efficacy from effectiveness studies. J Clin Epidemiol. 2006;59(10):1040-1048. doi:http://dx.doi.org/10.1016/j. jclinepi.2006.01.011.

49. Wu CY, Chang CK, Robson D, et al. Evaluation of smoking status identification using electronic health records and open-text information in a large mental health case register. PLoS One. 2013;8(9):e74262. doi:10.1371/ journal.pone.0074262.

50. Saw A, Kim J, Lim J, Powell C, Tong EK. Smoking cessation counseling for Asian immigrants with serious mental illness: using RE-AIM to understand challenges and lessons learned in primary care-behavioral health integration. Health Promot Pract. 2013;14(5 suppl):70S-79S. doi:10.1177/1524839913483141.

51. Peckham E, Man MS, Mitchell N, et al. Smoking Cessation Intervention for severe Mental Ill Health Trial (SCIMITAR): a pilot randomised control trial of the clinical effectiveness and cost-effectiveness of a bespoke smoking cessation service. Health Technol Assess. 2015;19(25):1-148. .doi:10.3310/hta19250.

52. Keenan TE, Yu A, Cooper LA, et al. Racial patterns of cardiovascular disease risk factors in serious mental illness and the overall U.S. population. Schizophr Res. 2013;150(1):211-216. doi:10.1016/j. schres.2013.07.022.

53. IBM Corp. IBM SPSS Statistics for Windows, Version 22.0. Armonk, NY: IBM Corp.; 2013.

54. Agresti A. Inference for contingency tables. Categorical Data Analysis. 2nd ed. New York, NY: John Wiley \& Sons, Inc; 2002:70-114.

55. Public Health Research Advisory Committee. Report of the Review of Public Health Research Funding in Australia. Canberra, Australia: National Health and Medical Research Council; 2009.

56. Williams JM, Steinberg ML, Griffiths KG, Cooperman N. Smokers with behavioral health comorbidity should be designated a tobacco use disparity group. Am J Public Health. 2013;103(9):1549-1555. doi:10.2105/ AJPH.2013.301232.

57. Fiore MC, Jaen CR, Baker TB. Treating Tobacco Use and Dependence: 2008 Update. Clinical Practice Guideline. Rockville, MD: U.S. Department of Health and Human Services: Public Health Service; 2008.

58. Hall SM, Prochaska JJ. Treatment of smokers with co-occurring disorders: emphasis on integration in mental health and addiction treatment settings. Annu Rev Clin Psychol. 2009;5(1):409-431. doi:10.1146/annurev. clinpsy.032408.153614.

59. Fakhoury W, Priebe S. Deinstitutionalization and reinstitutionalization: major changes in the provision of mental healthcare. Psychiatry. 2007;6(8):313-316. doi:http://dx.doi.org/10.1016/j.mppsy.2007.05.008.

60. Whiteford H, McKeon G, Harris M, et al. System-level intersectoral linkages between the mental health and non-clinical support sectors: a qualitative systematic review. Aust N Z J Psychiatry. 2014;48(10):895-906. doi:10.1177/0004867414541683.

61. Diaz F, Rendon D, Velasquez D, Susce M, Leon JD. Datapoints: smoking and smoking cessation among persons with severe mental illnesses. Psychiatr Serv. 2006;57(4):462. 